Pacific

Journal of

Mathematics

A SHORT PROOF OF THE EXISTENCE OF SUPERCUSPIDAL REPRESENTATIONS FOR ALL REDUCTIVE $p$-ADIC GROUPS

RAPHAËL BEUZART-PLESSIS 


\title{
A SHORT PROOF OF THE EXISTENCE OF SUPERCUSPIDAL REPRESENTATIONS FOR ALL REDUCTIVE $p$-ADIC GROUPS
}

\author{
RAPHAËL BEUZART-PLESSIS
}

\begin{abstract}
Let $G$ be a reductive $p$-adic group. We give a short proof of the fact that $G$ always admits supercuspidal complex representations. This result has already been established by A. Kret using the Deligne-Lusztig theory of representations of finite groups of Lie type. Our argument is of a different nature and is self-contained. It is based on the Harish-Chandra theory of cusp forms and it ultimately relies on the existence of elliptic maximal tori in $\boldsymbol{G}$.
\end{abstract}

Let $p$ be a prime number and let $F$ be a $p$-adic field (i.e., a finite extension of $\mathbb{Q}_{p}$ ). We denote by 0 the ring of integers of $F$ and we fix a uniformizer $\varpi \in \mathbb{O}$. We also denote by val $: F^{\times} \rightarrow \mathbb{Z}$ the normalized valuation (i.e., $\left.\operatorname{val}(\varpi)=1\right)$. Let $G$ be a connected reductive group defined over $F$. We will denote by $\mathfrak{g}$ the Lie algebra of $G$. A sentence like "Let $P=M N$ be a parabolic subgroup of $G$ " will mean as usual that $P$ is a parabolic subgroup of $G$ defined over $F$, that $N$ is its unipotent radical and that $M$ is a Levi component of $P$ also defined over $F$. More generally, all subgroups of $G$ that we consider will be defined over $F$. We will also need to fix Haar measures on the various groups that we consider. The precise normalization of these Haar measures won't be important (unless we specify that they need to satisfy an explicit compatibility condition) and we will only make use of Haar measures on unimodular groups (e.g., $F$ points of reductive or unipotent groups), so that the distinction between left and right Haar measures is irrelevant here and will be dropped from the notations.

Remark 1. We exclude fields of positive characteristic because we will use in a crucial way the exponential map. If $G=\mathrm{GL}_{n}$, then we can instead use the map $X \mapsto \mathrm{Id}+X$ and work over any nonarchimedean local field. For classical groups,

This work was supported by the Gould Fund and by the National Science Foundation under agreement No. DMS-1128155. Any opinions, findings and conclusions or recommendations expressed in this material are those of the author and do not necessarily reflect the views of the National Science Foundation.

MSC2010: primary 22E50; secondary 11F85.

Keywords: $p$-adic groups, supercuspidal representations, cusp forms. 
we could probably also replace the exponential map by some Cayley map and considerably weaken the characteristic assumption.

Recall that a smooth representation of $G(F)$ is a pair $\left(\pi, V_{\pi}\right)$ where $V_{\pi}$ is a complex vector space (usually infinite dimensional) and $\pi: G(F) \rightarrow \mathrm{GL}\left(V_{\pi}\right)$ is

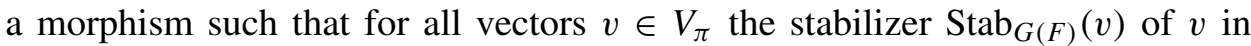
$G(F)$ is an open subgroup. Let $\left(\pi, V_{\pi}\right)$ be a smooth representation of $G(F)$ and let $P=M N$ be a parabolic subgroup of $G$. The Jacquet module of $\left(\pi, V_{\pi}\right)$ with respect to $P$ is the space of coinvariants

$$
V_{\pi, N}=V_{\pi} / V_{\pi}(N),
$$

where $V_{\pi}(N)$ is the subspace of $V_{\pi}$ generated by the elements $v-\pi(n) v$ for all $v \in V_{\pi}$ and all $n \in N(F)$. It is the biggest quotient of $V_{\pi}$ on which $N(F)$ acts trivially. There is a natural linear action $\pi_{N}$ of $M(F)$ on $V_{\pi, N}$ and $\left(\pi_{N}, V_{\pi, N}\right)$ is a smooth representation of $M(F)$. The functor $V_{\pi} \mapsto V_{\pi, N}$ is an exact functor from the category of smooth representations of $G(F)$ to the category of smooth representations of $M(F)$. Indeed, this follows rather easily from the following fact (see [Renard 2010, Proposition III.2.9]):

(1) Let $\left(N(F)_{k}\right)_{k \geqslant 0}$ be an increasing sequence of compact-open subgroups of $N(F)$ such that $N(F)=\bigcup_{k \geqslant 0} N(F)_{k}$ (such a sequence always exists). Then a vector $v \in V_{\pi}$ belongs to $V_{\pi}(N)$ if and only if there exists $k \geqslant 0$ such that

$$
\int_{N(F)_{k}} \pi(n) v d n=0 .
$$

Let $\left(\pi, V_{\pi}\right)$ be an irreducible smooth representation of $G(F)$ (irreducible means that $V_{\pi}$ is nontrivial and that it has no nonzero proper $G(F)$-invariant subspace). The representation $\left(\pi, V_{\pi}\right)$ is said to be supercuspidal if for all proper parabolic subgroups $P=M N$ of $G$, the Jacquet module $V_{\pi, N}$ is zero. An equivalent condition is that the coefficients of $\left(\pi, V_{\pi}\right)$ are compactly supported modulo the center (see [Renard 2010, Theorem VI.2.1]).

The purpose of this short note is to prove the following result.

Theorem 2. $G(F)$ admits irreducible supercuspidal representations.

Remark 3. This theorem has already been proved by A. Kret [2012]. The proof of Kret has the advantage of working in any characteristic (cf. Remark 1) and of being explicit (i.e., it exhibits a way to construct such supercuspidal representations by compact induction). Kret's proof eventually relies on the Deligne-Lusztig theory of representations of finite groups of Lie type and so can hardly be considered elementary. Although less complete and explicit than the results of [Kret 2012], the proof presented here has the advantage of being short and (almost) self-contained, using only elementary harmonic analysis arguments. 
We will deduce Theorem 2 from the existence of nonzero compactly supported cusp forms, in the sense of Harish-Chandra, for the group $G(F)$. Before stating this existence result, we need to introduce some more definitions and notation. We will denote, as usual, by $C_{c}^{\infty}(G(F))$ the space of complex-valued functions on $G(F)$ that are smooth, i.e., locally constant, and compactly supported. We say that a function $f \in C_{c}^{\infty}(G(F))$ is a cusp form if for all proper parabolic subgroups $P=M N$ of $G$, we have

$$
\int_{N(F)} f(x n) d n=0, \quad \text { for all } x \in G(F)
$$

(these functions are called supercusp forms in [Harish-Chandra 1970]). We shall denote by $C_{c \text {,cusp }}^{\infty}(G(F)) \subseteq C_{c}^{\infty}(G(F))$ the subspace of cusp forms. As we said, Theorem 2 will follow from the following existence theorem.

Theorem 4. We have $C_{c, \text { cusp }}^{\infty}(G(F)) \neq 0$.

Proof that Theorem 4 implies Theorem 2. Let us denote by $\rho$ the action of $G(F)$ on $C_{c}^{\infty}(G(F))$ by right translation. Then $\left(\rho, C_{c}^{\infty}(G(F))\right)$ is a smooth representation of $G(F)$. Moreover, it is easy to see that the subspace $C_{c \text {,cusp }}^{\infty}(G(F)) \subseteq C_{c}^{\infty}(G(F))$ is $G(F)$-invariant. We claim the following:

(2) For all proper parabolic subgroups $P=M N$ of $G$, we have

$$
C_{c, \text { cusp }}^{\infty}(G(F))_{N}=0 .
$$

Let $P=M N$ be a proper parabolic subgroup of $G$ and fix an increasing sequence $\left(N(F)_{k}\right)_{k \geqslant 0}$ of compact-open subgroups of $N(F)$ such that $N(F)=\bigcup_{k \geqslant 0} N(F)_{k}$. Let $f \in C_{c \text {,cusp }}^{\infty}(G(F))$. By (1), it suffices to show the existence of an integer $k \geqslant 0$ such that

$$
\int_{N(F)_{k}} \rho(n) f d n=0
$$

or, what amounts to the same,

$$
\int_{N(F)_{k}} f(x n) d n=0, \quad \text { for all } x \in G(F) .
$$

Since $\operatorname{Supp}(f)$ (the support of the function $f$ ) is compact, there exists $k \geqslant 0$ such that

$$
\operatorname{Supp}(f) \cap\left[\operatorname{Supp}(f)\left(N(F) \backslash N(F)_{k}\right)\right]=\varnothing .
$$

We now show that (3) is satisfied for such $k$. Let $x \in G(F)$. If $x \notin \operatorname{Supp}(f) N(F)_{k}$, the term inside the integral (3) vanishes identically and there is nothing to prove. Assume from now on that $x \in \operatorname{Supp}(f) N(F)_{k}$. Up to translating $x$ by an element 
of $N(F)_{k}$, we may as well assume that $x \in \operatorname{Supp}(f)$. Then, by (4) we have $x n \notin \operatorname{Supp}(f)$ for all $n \in N(F) \backslash N(F)_{k}$. It follows that

$$
\int_{N(F)_{k}} f(x n) d n=\int_{N(F)} f(x n) d n .
$$

But by definition of $C_{c \text {,cusp }}^{\infty}(G(F))$, this last integral vanishes. This proves (3) and ends the proof of (2).

We now show how to deduce from (2) that Theorem 4 implies Theorem 2. Assume that Theorem 4 is satisfied. Then, we can find $f \in C_{c, \text { cusp }}^{\infty}(G(F))$ which is nonzero. Denote by $V_{f}$ the $G(F)$-invariant subspace of $C_{c, \text { cusp }}^{\infty}(G(F))$ generated by $f$ and let $V \subseteq V_{f}$ be a maximal $G(F)$-invariant subspace among those not containing $f$ (which exists by Zorn's lemma). Then $V_{f} / V$ is a smooth irreducible representation of $G(F)$. We claim that it is supercuspidal. Indeed, let $P=M N$ be a proper parabolic subgroup of $G$. By (2) and since the Jacquet module's functor is exact, we have $\left(V_{f} / V\right)_{N}=0$. Thus, $V_{f} / V$ is indeed a supercuspidal representation of $G(F)$ and this proves Theorem 2 .

We are now left with proving Theorem 4. The strategy is to prove first an analog result on the Lie algebra and then lift it to the group by means of the exponential map. Let $C_{c}^{\infty}(\mathfrak{g}(F))$ be the space of complex-valued smooth and compactly supported functions on $\mathfrak{g}(F)$. We say that a function $\varphi \in C_{c}^{\infty}(\mathfrak{g}(F))$ is a cusp form if for all proper parabolic subgroups $P=M N$ of $G$, we have

$$
\int_{\mathfrak{n}(F)} \varphi(X+N) d n=0, \quad \text { for all } X \in \mathfrak{g}(F),
$$

where $\mathfrak{n}(F)$ denotes the $F$-points of the Lie algebra of $N$. We will denote by $C_{c, \text { cusp }}^{\infty}(\mathfrak{g}(F)) \subseteq C_{c}^{\infty}(\mathfrak{g}(F))$ the subspace of cusp forms. The analog of Theorem 4 for the Lie algebra is the following lemma.

Lemma 5. We have $C_{c, \text { cusp }}^{\infty}(\mathfrak{g}(F)) \neq 0$.

Before proving this lemma, we first explain how it implies Theorem 4.

Proof that Lemma 5 implies Theorem 4. Assume that Lemma 5 holds. Then, we can find a nonzero function $\varphi \in C_{c, \text { cusp }}^{\infty}(\mathfrak{g}(F))$. The idea is to lift $\varphi$ to a function on $G(F)$ using the exponential map. Of course, the exponential map is not necessarily defined on the support of $\varphi$. Hence, we need first to scale the function $\varphi$ so that its support becomes small. Let us fix an element $\lambda \in F^{\times}$which we will eventually assume to be sufficiently small. We define the function $\varphi_{\lambda}$ by

$$
\varphi_{\lambda}(X)=\varphi\left(\lambda^{-1} X\right), \quad X \in \mathfrak{g}(F) .
$$

We easily check that $\varphi_{\lambda}$ is still a cusp form. Recall that there exists an open neighborhood $\omega \subseteq \mathfrak{g}(F)$ of 0 on which the exponential map exp is defined and such 
that it realizes an $F$-analytic isomorphism

$$
\exp : \omega \simeq \Omega,
$$

where $\Omega=\exp (\omega)$. Since $\operatorname{Supp}\left(\varphi_{\lambda}\right)=\lambda \operatorname{Supp}(\varphi)$, for $\lambda$ sufficiently small we have

$$
\operatorname{Supp}\left(\varphi_{\lambda}\right) \subseteq \omega .
$$

We henceforth assume that $\lambda$ is that sufficiently small. This allows us to define a function $f_{\lambda}$ on $G(F)$ by setting

$$
f_{\lambda}(g)= \begin{cases}\varphi_{\lambda}(X) & \text { if } g=\exp (X) \text { for some } X \in \omega, \\ 0 & \text { otherwise }\end{cases}
$$

for all $g \in G(F)$. Note that we have $f_{\lambda} \in C_{c}^{\infty}(G(F))$, and obviously the function $f_{\lambda}$ is nonzero. Hence, we will be done if we can prove the following:

(5) If $\lambda$ is sufficiently small, the function $f_{\lambda}$ is a cusp form.

Let us denote by $\log : \Omega \rightarrow \omega$ the inverse of the exponential map. Then, by the Campbell-Hausdorff formula, it is easy to see that we can find an O-lattice $L$ in the $F$-vector space $\mathfrak{g}(F)$ which is contained in $\omega$ and satisfies the following condition:

$$
\log \left(e^{X} e^{Y}\right) \in X+Y+\varpi^{\operatorname{val}_{L}(X)+\operatorname{val}_{L}(Y)} L
$$

for all $X, Y \in L$, where we have set $\operatorname{val}_{L}(X)=\sup \left\{k \in \mathbb{Z}: X \in \varpi^{k} L\right\}$ for all $X \in \mathfrak{g}(F)$. For all integers $n \geqslant 0$, set $K_{n}=\exp \left(\varpi^{n} L\right)$. It is easy to infer from (6) that $K_{n}$ is an open-compact subgroup of $G(F)$ for all $n \geqslant 0$. Since $\varphi$ is smooth and compactly supported, there exists $n_{0} \geqslant 0$ such that translation by $\varpi^{n_{0}} L$ leaves $\varphi$ invariant. Also, since $\varphi$ is compactly supported, there exists $n_{1} \geqslant 0$ such that $\operatorname{Supp}(\varphi) \subseteq \varpi^{-n_{1}} L$. We will show that (5) holds provided $\operatorname{val}(\lambda) \geqslant 2 n_{1}+n_{0}$. Assume this is so and set $n=\operatorname{val}(\lambda)-n_{1}$. Then we have

$$
\operatorname{Supp}\left(\varphi_{\lambda}\right)=\lambda \operatorname{Supp}(\varphi) \subseteq \lambda \varpi^{-n_{1}} L=\varpi^{n} L .
$$

Hence, it follows that

$$
\operatorname{Supp}\left(f_{\lambda}\right) \subseteq K_{n} .
$$

Let $P=M N$ be a proper parabolic subgroup of $G$ and let $x \in G(F)$. Consider the integral

$$
\int_{N(F)} f_{\lambda}(x n) d n
$$

If $x N(F) \cap K_{n}=\varnothing$, then by (8) the term inside the integral above vanishes identically and it follows that the integral is equal to zero. Assume from now on that $x K_{n} \cap N(F) \neq \varnothing$. Up to translating $x$ by an element of $N(F)$, we may assume that $x \in K_{n}$. Then we may write $x=e^{X}$ for some $X \in \varpi^{n} L$. Using (8) again, 
and since $K_{n}$ is a subgroup of $G(F)$, we see that the integral (9) is supported on $K_{n} \cap N(F)$. Thus, we have equalities

$$
\int_{N(F)} f_{\lambda}(x n) d n=\int_{K_{n} \cap N(F)} f_{\lambda}\left(e^{X} n\right) d n .
$$

Set $L_{N}=L \cap \mathfrak{n}(F)$. Then if we normalize measures correctly, the exponential map induces a measure preserving isomorphism $\varpi^{n} L_{N} \simeq K_{n} \cap N(F)$, so that we have

$$
\int_{K_{n} \cap N(F)} f_{\lambda}\left(e^{X} n\right) d n=\int_{\varpi^{n} L_{N}} f_{\lambda}\left(e^{X} e^{N}\right) d n=\int_{\varpi^{n} L} \varphi_{\lambda}\left(\log \left(e^{X} e^{N}\right)\right) d n .
$$

By (6), for all $N \in \varpi^{n} L_{N}$ we have

$$
\log \left(e^{X} e^{N}\right) \in X+N+\varpi^{2 n} L .
$$

Moreover, as $\varphi$ is invariant by translation by $\varpi^{n_{0}} L$, the function $\varphi_{\lambda}$ is invariant by translation by $\lambda \varpi^{n_{0}} L=\varpi^{n+n_{1}+n_{0}} L$ (recalling that $n=\operatorname{val}(\lambda)-n_{1}$ ). Since $\operatorname{val}(\lambda) \geqslant 2 n_{1}+n_{0}$, we also have $n \geqslant n_{1}+n_{0}$. Hence, the function $\varphi_{\lambda}$ is invariant by translation by $\varpi^{2 n} L$ and so using (12), we deduce

$$
\varphi_{\lambda}\left(\log \left(e^{X} e^{N}\right)\right)=\varphi_{\lambda}(X+N)
$$

for all $N \in \varpi^{n} L_{N}$. From (10) and (11), it follows that

$$
\int_{N(F)} f_{\lambda}(x n) d n=\int_{\varpi^{n} L} \varphi_{\lambda}(X+N) d n .
$$

By (7) and since $X \in \varpi^{n} L$, the function $N \mapsto \varphi_{\lambda}(X+N)$ for $N \in \mathfrak{n}(F)$ is supported on $\varpi^{n} L_{N}$. Consequently, we have

$$
\int_{\varpi^{n} L} \varphi_{\lambda}(X+N) d n=\int_{\mathfrak{n}(F)} \varphi_{\lambda}(X+N) d n .
$$

As $\varphi_{\lambda}$ is a cusp form, this last integral vanishes. Hence, using (13) we see that the integral (9) is also zero. Since it is true for all $x \in G(F)$ and all proper parabolic subgroups $P=M N$ of $G$, this shows that $f_{\lambda}$ is a cusp form. Hence, (5) is indeed satisfied as soon as $\operatorname{val}(\lambda) \geqslant 2 n_{1}+n_{0}$, and this ends the proof that Lemma 5 implies Theorem 4.

It only remains to establish Lemma 5. Recall that a maximal torus $T$ in $G$ is said to be elliptic if $A_{T}=A_{G}$, where $A_{T}$ and $A_{G}$ denote the maximal split subtori in $T$ and the center of $G$, respectively. The proof of Lemma 5 will ultimately rely on the following existence result (see [Platonov and Rapinchuk 1994, Theorem 6.21]):

Theorem 6. $G$ admits an elliptic maximal torus. 
Proof of Lemma 5. Let us fix a symmetric nondegenerate bilinear form $B$ on $\mathfrak{g}(F)$ which is $G(F)$-invariant. Such a bilinear form is easy to construct. On $\mathfrak{g}_{\text {der }}(F)$, the derived subalgebra of $\mathfrak{g}(F)$, we have the Killing form $B_{\text {Kil }}$ which is symmetric, $G(F)$-invariant and nondegenerate. Hence, we may take $B=B_{\mathfrak{z}} \oplus B_{\text {Kil }}$ where $B_{\mathfrak{z}}$ is any nondegenerate symmetric bilinear form on $\mathfrak{z}_{G}(F)$, the center of $\mathfrak{g}(F)$. Let us also fix a nontrivial continuous additive character $\psi: F \rightarrow \mathbb{C}^{\times}$. Using those, we can define a Fourier transform on $C_{c}^{\infty}(\mathfrak{g}(F))$ by

$$
\hat{\varphi}(Y)=\int_{\mathfrak{g}(F)} \varphi(X) \psi(B(X, Y)) d X, \quad \varphi \in C_{c}^{\infty}(\mathfrak{g}(F)), Y \in \mathfrak{g}(F) .
$$

Of course, this Fourier transform also depends on the choice of a Haar measure on $\mathfrak{g}(F)$.

More generally, if $V$ is a subspace of $\mathfrak{g}(F)$ and $V^{\perp}$ denotes the orthogonal of $V$ with respect to $B$, we can also define a Fourier transform $C_{c}^{\infty}(V) \rightarrow C_{c}^{\infty}\left(\mathfrak{g}(F) / V^{\perp}\right)$, $\varphi \mapsto \hat{\varphi}$, by setting

$$
\hat{\varphi}(Y)=\int_{V} \varphi(Y) \psi(B(X, Y)) d Y, \quad X \in \mathfrak{g}(F) / V^{\perp},
$$

where again we need to choose a Haar measure on $V$. It is easy to check that for compatible choices of Haar measures, the following diagram commutes:

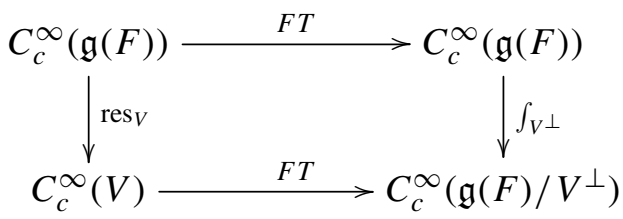

whereby the horizontal arrows are Fourier transforms, the left vertical arrow is given by restriction to $V$ and the right vertical arrow is given by integration over the cosets of $V^{\perp}$. For $P=M N$ a parabolic subgroup of $G$, we have $\mathfrak{p}(F)^{\perp}=\mathfrak{n}(F)$ (where $\mathfrak{p}$ stands for the Lie algebra of $P$ ). The commutativity of the above diagram in this particular case gives us (for some compatible choices of Haar measures) the formula

$$
\int_{\mathfrak{n}(F)} \hat{\varphi}(X+N) d n=\int_{\mathfrak{p}(F)} \varphi(Y) \psi(B(X, Y)) d Y
$$

for all $\varphi \in C_{c}^{\infty}(\mathfrak{g}(F))$ and all $X \in \mathfrak{g}(F)$.

Let $T_{\text {ell }}$ be an elliptic maximal torus of $G$, whose existence is guaranteed by Theorem 6. Let $\mathfrak{t}_{\text {ell }}$ be its Lie algebra and set $\mathfrak{t}_{\text {ell,reg }}=\mathfrak{t}_{\text {ell }} \cap \mathfrak{g}_{\text {reg }}$ for the subset of $G$-regular elements in $\mathfrak{t}_{\text {ell }}$. Denote by $\mathfrak{t}_{\text {ell,reg }}(F)^{G}$ the subset of elements in $\mathfrak{g}_{\text {reg }}(F)$ that are $G(F)$-conjugated to an element of $\mathfrak{t}_{\text {ell, reg }}(F)$. Then, $\mathfrak{t}_{\text {ell, reg }}(F)^{G}$ is an open subset of $\mathfrak{g}(F)$ (since the map $T_{\text {ell }}(F) \backslash G(F) \times \mathfrak{t}_{\text {ell, reg }}(F) \rightarrow \mathfrak{g}(F)$, 
$(g, X) \mapsto g^{-1} X g$, is everywhere submersive). In particular, we can certainly find a nonzero function $\varphi \in C_{c}^{\infty}(\mathfrak{g}(F))$ whose support is contained in $\mathfrak{t}_{\text {ell, reg }}(F)^{G}$. Let us fix such a function $\varphi$. We claim the following:

(15) The function $\hat{\varphi}$ is a cusp form.

Indeed, let $P=M N$ be a proper parabolic subgroup of $G$ and let $X \in \mathfrak{g}(F)$. We need to see that the integral

$$
\int_{\mathfrak{n}(F)} \hat{\varphi}(X+N) d n
$$

is zero. By (14), this integral is equal to

$$
\int_{\mathfrak{p}(F)} \varphi(Y) \psi(B(X, Y)) d Y .
$$

Hence, we only need to show that $\operatorname{Supp}(\varphi) \cap \mathfrak{p}(F)=\varnothing$. By definition of $\varphi$, it even suffices to see that $\mathfrak{t}_{\text {ell,reg }}(F)^{G} \cap \mathfrak{p}(F)=\varnothing$. But this follows immediately from the fact that, $P$ being proper, it does not contain any elliptic maximal torus of $G$.

\section{References}

[Harish-Chandra 1970] Harish-Chandra, Harmonic analysis on reductive p-adic groups, Lecture Notes in Mathematics 162, Springer, Berlin, 1970. MR 54 \#2889 Zbl 0202.41101

[Kret 2012] A. Kret, "Existence of cuspidal representations of $p$-adic reductive groups", preprint, 2012. arXiv 1205.2771

[Platonov and Rapinchuk 1994] V. Platonov and A. Rapinchuk, Algebraic groups and number theory, Pure and Applied Mathematics 139, Academic Press, Boston, 1994. MR 95b:11039 Zbl 0841.20046 [Renard 2010] D. Renard, Représentations des groupes réductifs p-adiques, Cours Spécialisés 17, Société Mathématique de France, Paris, 2010. MR 2011d:22019 Zbl 1186.22020

Received June 9, 2015. Revised August 17, 2015.

RAPHAËL BEUZART-PLESSIS

DEPARTMENT OF MATHEMATICS

NATIONAL UNIVERSITY OF SINGAPORE

BLOCK 17, 10 LOWER KENT RIDGE ROAD

SINGAPORE 119076

SINGAPORE

rbeuzart@gmail.com 


\title{
PACIFIC JOURNAL OF MATHEMATICS
}

\author{
msp.org/pjm
}

Founded in 1951 by E. F. Beckenbach (1906-1982) and F. Wolf (1904-1989)

\section{EDITORS}

Don Blasius (Managing Editor)

Department of Mathematics

University of California

Los Angeles, CA 90095-1555

blasius@math.ucla.edu

\author{
Paul Balmer \\ Department of Mathematics \\ University of California \\ Los Angeles, CA 90095-1555 \\ balmer@math.ucla.edu \\ Robert Finn \\ Department of Mathematics \\ Stanford University \\ Stanford, CA 94305-2125 \\ finn@math.stanford.edu \\ Sorin Popa \\ Department of Mathematics \\ University of California \\ Los Angeles, CA 90095-1555 \\ popa@math.ucla.edu
}

\author{
Vyjayanthi Chari \\ Department of Mathematics \\ University of California \\ Riverside, CA 92521-0135 \\ chari@math.ucr.edu \\ Kefeng Liu \\ Department of Mathematics \\ University of California \\ Los Angeles, CA 90095-1555 \\ liu@math.ucla.edu \\ Jie Qing \\ Department of Mathematics \\ University of California \\ Santa Cruz, CA 95064 \\ qing@ cats.ucsc.edu
}

\section{PRODUCTION}

Silvio Levy, Scientific Editor, production@msp.org

\section{SUPPORTING INSTITUTIONS}

ACADEMIA SINICA, TAIPEI

CALIFORNIA INST. OF TECHNOLOGY

INST. DE MATEMÁTICA PURA E APLICADA

KEIO UNIVERSITY

MATH. SCIENCES RESEARCH INSTITUTE

NEW MEXICO STATE UNIV.

OREGON STATE UNIV.

\author{
STANFORD UNIVERSITY \\ UNIV. OF BRITISH COLUMBIA \\ UNIV. OF CALIFORNIA, BERKELEY \\ UNIV. OF CALIFORNIA, DAVIS \\ UNIV. OF CALIFORNIA, LOS ANGELES \\ UNIV. OF CALIFORNIA, RIVERSIDE \\ UNIV. OF CALIFORNIA, SAN DIEGO \\ UNIV. OF CALIF., SANTA BARBARA
}

\author{
Daryl Cooper \\ Department of Mathematics \\ University of California \\ Santa Barbara, CA 93106-3080 \\ cooper@math.ucsb.edu \\ Jiang-Hua Lu \\ Department of Mathematics \\ The University of Hong Kong \\ Pokfulam Rd., Hong Kong \\ jhlu@maths.hku.hk \\ Paul Yang \\ Department of Mathematics \\ Princeton University \\ Princeton NJ 08544-1000 \\ yang@math.princeton.edu
}

These supporting institutions contribute to the cost of publication of this Journal, but they are not owners or publishers and have no responsibility for its contents or policies.

See inside back cover or msp.org/pjm for submission instructions.

The subscription price for 2016 is US $\$ 440 /$ year for the electronic version, and $\$ 600 /$ year for print and electronic.

Subscriptions, requests for back issues and changes of subscribers address should be sent to Pacific Journal of Mathematics, P.O. Box 4163, Berkeley, CA 94704-0163, U.S.A. The Pacific Journal of Mathematics is indexed by Mathematical Reviews, Zentralblatt MATH, PASCAL CNRS Index, Referativnyi Zhurnal, Current Mathematical Publications and Web of Knowledge (Science Citation Index).

The Pacific Journal of Mathematics (ISSN 0030-8730) at the University of California, c/o Department of Mathematics, 798 Evans Hall \#3840, Berkeley, CA 94720-3840, is published twelve times a year. Periodical rate postage paid at Berkeley, CA 94704, and additional mailing offices. POSTMASTER: send address changes to Pacific Journal of Mathematics, P.O. Box 4163, Berkeley, CA 94704-0163.

PJM peer review and production are managed by EditFLOW ${ }^{\circledR}$ from Mathematical Sciences Publishers.

\section{PUBLISHED BY}

\section{mathematical sciences publishers \\ nonprofit scientific publishing}

http://msp.org/

(C) 2016 Mathematical Sciences Publishers 


\section{PACIFIC JOURNAL OF MATHEMATICS}

Volume 282 No. $1 \quad$ May 2016

On the half-space theorem for minimal surfaces in Heisenberg space

Tristan Alex

Extending smooth cyclic group actions on the Poincaré homology sphere 9

NIMA ANVARI

A short proof of the existence of supercuspidal representations for all reductive $p$-adic groups

RAPHAËL BEUZART-PLESSIS

Quantum groups and generalized circular elements

MICHAEL BRANNAN and KAY KIRKPATRICK

Volumes of Montesinos links

KathleEn Finlinson and Jessica S. PurCell

Minimal surfaces with two ends which have the least total absolute curvature

SHOICHI FUJIMORI and TOSHIHIRO SHODA

Multiplicité du spectre de Steklov sur les surfaces et nombre chromatique

\section{PIERRE JAMMES}

$E$-polynomial of the $\mathrm{SL}(3, \mathbb{C})$-character variety of free groups

SEAN LAWTON and VicEnTE MuÑOZ

The Blum-Hanson property for $\mathscr{C}(K)$ spaces

PASCAL LEFÈVRE and ÉTIENNE MATHERON

Crossed product algebras and direct integral decomposition for Lie supergroups

KARL-HERMANN NEEB and HADI SALMASIAN Associated primes of local cohomology modules over regular rings

TONY J. PUTHENPURAKAL 